\title{
An Efficient Scheme for Broadband Adaptive Beamforming
}

\author{
Stephan Weiss ${ }^{1}$, Robert W. Stewart ${ }^{2}$, Marion Schabert ${ }^{2}$, Ian K. Proudler ${ }^{3}$. \\ and Michael W. Hoffman ${ }^{4}$ \\ ${ }^{1}$ Dept. Electronics \& Computer Science, University of Southampton, UK \\ ${ }^{2}$ Dept. Electronic \& Electrical Eng.. University of Strathclyde, Glasgow, Scotland, UK \\ ${ }^{3}$ Defence Evaluation and Research Agency; Malvern, UK \\ ${ }^{4}$ Dept. Electrical Engineering, University of Nebraska. Lincoln, NE \\ s.weiss@ecs.soton.ac.uk, r.stewart@eee.strath.ac.uk
}

\begin{abstract}
This paper introduces an oversampled subband approach to linearly constrained minimum variance adaptive broadband beamforming. This method is motivated by the considerable reduction in computation over fullband implementation and resulting large computational complexity when fullband beamformers with high spatial and spectral resolution are required. We present the proposed subband adaptive beamformer structure, discuss advantages and limitations of it, and comment on the correct projection of the constraints in the subband domain. In a simulation, the proposed subband structure is compared to a fullband adaptive beamformer, highlighting the benefit of our method.
\end{abstract}

\section{Introduction}

In beamforming, the task is to steer a sensor array towards a signal impinging from a certain direction, while interfering signals from other spatial angles of incident are suppressed [2. 9]. Fig. 1 shows a broadband beamformer, where each discretely sampled sensor signal $x_{m}[n]$ is processed by a filter with coefficients organized in a vector $\mathbf{w}_{m}$. If the wavefronts of a signal impinging from an angle $\vartheta$ arrive delayed by integer multiples of $\Delta \tau$ at the $M$ sensors, the filters $\mathbf{w}_{m}$ should re-align the wavefronts by implementing the appropriate (generally fractional) delays.

To perform beamforming at high spatial resolution, generally FIR filters of considerable length are required to accurately match fractioral delays [8] which are necessary to align the differently delayed signals at the summation point in Fig. 1 for constructive or destructive interference. The resulting large complexity has motivated efficient computational methods, such as processing in the frequency domain [1]. In this paper, we want to evaluate subband techniques, whereby filtering is performed in decimated frequency bands $[7,3]$.

In the following, we first review linearly constrained minimum variance broadband beamformers in Sec. 2 . In Sec. 3 we introduce the structure, components, and constraint design of the proposed subband adaptive beamformer $(\mathrm{SAB})$. Simulations and comparisons of the $\mathrm{SAB}$ to a fullband implementation are given in Sec. 4 .

\section{LCMV Beamforming}

This section will provide a summary of adaptive linearly constrained minimum variance (LCMV) beamformers, in particular a version known as the generalized sidelobe canceller. Of particular interest will be the selection of constraints.

\subsection{Structure and Formulation}

Fig. 1 introduced the structure of a general beamformer. For notational convenience, the coefficients of the beamforming filters $\mathbf{w}_{m}, m=0(1) M-1$, are col-

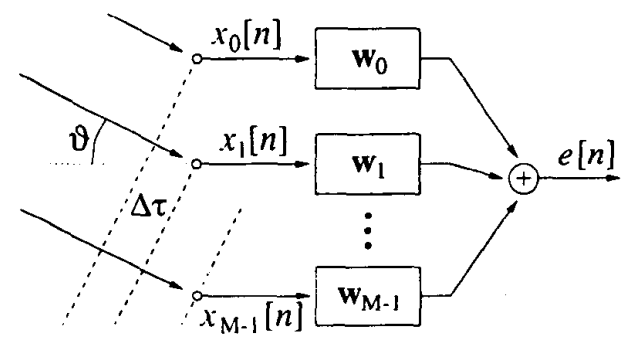

Fig. 1. Broadband beamformer. 
lected in a single vector $\mathbf{w}$,

$$
\mathbf{w}=\left[\begin{array}{c}
\mathbf{w}_{0} \\
\mathbf{w}_{1} \\
\vdots \\
\mathbf{w}_{M-1}
\end{array}\right]
$$

With a similar definition of a vector $\mathbf{x}[n]$ which contains all sensor input samples in the delay line of the filters $\mathbf{w}_{m}[n]$, the beamformer output $e[n]$ can be written as

$$
e[n]=\mathbf{w}^{H} \mathbf{x}[n] .
$$

The LCMV problem is then given by

$$
\begin{aligned}
\mathbf{w}_{\mathrm{opt}}= & \arg \min _{\mathbf{w}} \mathbf{w}^{H} \mathbf{R}_{r x} \mathbf{w} \\
& \text { subject to } \quad \mathbf{C}^{H} \mathbf{w}=\mathbf{f},
\end{aligned}
$$

where $\mathbf{R}_{x x}$ is the covariance matrix built from the input $\mathbf{x}[n]$. $\mathbf{C}$ the constraint matrix, and $\mathbf{f}$ the constraining vector. The choice of $\mathbf{C}$ and $\mathbf{f}$ will be commented on in Sec. 2.3. An analytic optimum Wiener solution for (3) can be derived [9]. Iterative methods to determine $\mathbf{w}_{\text {opt }}$ have been suggested, and include the Frost beamformer [2], and the generalised sidelobe canceller (GSC [4]), which will be used in the following.

\subsection{Generalised Sidelobe Canceller}

The GSC performs an unconstrained optimization based on a projection of the input signal $\mathbf{x}[n]$ away from the constraint subspace,

$$
\mathbf{u}[n]=\mathbf{C}_{\mathbf{a}} \mathbf{x}[n] .
$$

The projection matrix $\mathbf{C}_{a}$, also known as blocking matrix, spans the nullspace of $\mathbf{C}^{H}$ and can be determined by. for example, singular value decomposition of the constraint matrix $\mathbf{C}$. The quiescent vector $\mathbf{w}_{q}=\mathbf{C}\left(\mathbf{C}^{H} \mathbf{C}\right)^{-1} \mathbf{f}$ defines a desired signal

$$
d[n]=\mathbf{w}_{q}^{H} \mathbf{x}[n],
$$

which fulfills the constraints but still contains interference terms. The resulting GSC structure with an adaptive coefficient vector $\mathbf{w}_{a}$ is shown in Fig. 2 .

The optimisation of the vector $\mathbf{w}_{a}$ is an unconstrained problem and can be solved using standard adaptive filters based on least mean square (LMS) type or recursive least squares (RLS) algorithms [5] to cancel the interference terms in $d[n]$. As an example, a GSC based on the normalised LMS (NLMS) algorithm would take the form

$$
\begin{aligned}
e[n] & =d[n]-\mathbf{w}_{a}^{H}[n] \cdot \mathbf{u}[n] \\
\mathbf{w}_{a}[n+1] & =\mathbf{w}_{a}[n]+\frac{\tilde{\mu}}{\|\mathbf{u}[n]\|_{2}^{2}} e^{*}[n] \cdot \mathbf{u}[n]
\end{aligned}
$$

Similarly, any other adaptive filter could be constructed based on the the input signal $u[n]$ and the desired signal $d[n]$.

\subsection{Constraint Design}

To correctly handle the constraint design in the subband case later, a brief consideration of the fullband case is given. interpreting the constraint definition as a convolution. If we assume that the signal of interest illuminating the array from broadside should be preserved, the constraint equation can be formulated as

$$
\underbrace{\left[\begin{array}{lll}
\mathbf{I}_{L} & \mathbf{I}_{L} \cdot \mathbf{I}_{L}
\end{array}\right]}_{\mathbf{C}^{H}} \cdot \mathbf{w}=\mathbf{f} .
$$

The constraining vector $\mathbf{f}$ determines the desired impulsc response of the array in the look direction:

$$
\mathbf{f}=\left[\begin{array}{llll}
f_{0} & f_{1} & \ldots & f_{L-1}
\end{array}\right]^{H} .
$$

For a reception indiscriminate of frequency, $\mathbf{f}$ could be a centred impulse. However, sometimes the signal of interest has spectral properties, which can be incorporated into the design [2]. Also, as the array exhibits almost no spatial resolution at very low frequencies. sometimes a highpass characterstic can be embedded into $f$ to improve the performance of the beamformer [6].

\section{Subband Adaptive Beamforming}

\subsection{Oversampled Subband Adaptive Filtering}

Subband adaptive filtering is popularly based on oversampled filter banks $[7,11]$, where the decimation rate $N$ is smaller than the number of frequency bands $K$. The decomposition into oversampled subbands is performed by analysis and synthesis filter banks as shown in Fig. 3. If the filter banks are chosen correctly; the desired and input signal usually supplied to a standard fullband adaptive filter can instead be

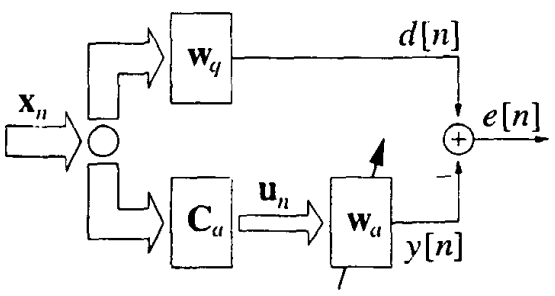

Fig. 2. Generalized sidelobe canceller. 


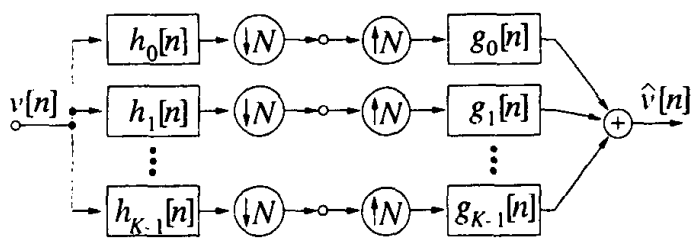

analysis filter bank synthesis filter bank

Fig. 3. Analysis and synthesis filter bank.

passed through analysis filter banks, and adaptive filters are applied independently in each subband. This is shown in Fig. 5 for an adaptive system identification setup. Finally, the resulting subband error signals can be reconstructed back into the fullband via the synthesis bank shown in Fig. 3.

Performing subband adaptive filtering (SAF) allows a reduced computational complexity due to the fact that (i) the filters operating in the subband domain can be shortened by a factor of $\approx N$ in order to achieve an identical modeling capability and (ii) the update rate is reduced by a factor of $N$. The analysis filter bank generally also achieves a whitening of the signals in the subband domain, which results in a potentially faster convergence for LMS-type algorithms. Besides the low processing cost in subbands, the filter bank operations can be implemented efficiently and memory saving by employing modulated filter banks [10], whereby all filters in Fig. 3 are derived from the same prototype filter by complex modulation. This also means that for real valued input signals, $K / 2$ subbands are sufficient to process, as the remaining subbands are only complex conjugate copies [11].

In the absence of other error sources such as observation noise or model truncation, the limitations of S.AF lie in the aliasing produced in the subbands, which limits the minimum achievable error, and in the crror in power complementarity of the filter banks, which sets the maximally achievable accuracy of the overall SAF structure. In the case of modulated filter banks, both errors can be stated in terms of the prototype filter [13].

\subsection{Subband Adaptive Beamforming Structure}

The proposed subband adaptive beamformer ( $\mathrm{SAB}$ ) structure decomposes each sensor signal $x_{m}[n]$ by means of an analysis filter bank, and applies an independent beamformer to each subband, as shown in Fig. 4. In this case, the subband beamforming algorithms are GSCs as given in Fig. 2, but could as well be replaced by other LCMV beamformers. Of importance however is the application of correct constraints

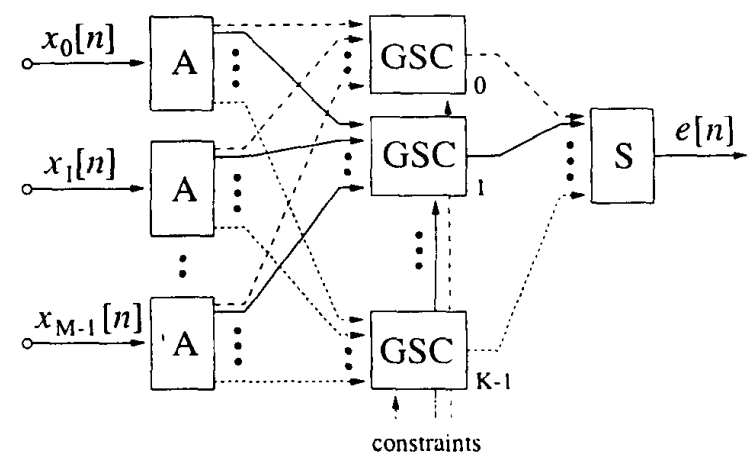

Fig. 4. Subband adaptive beamformer.

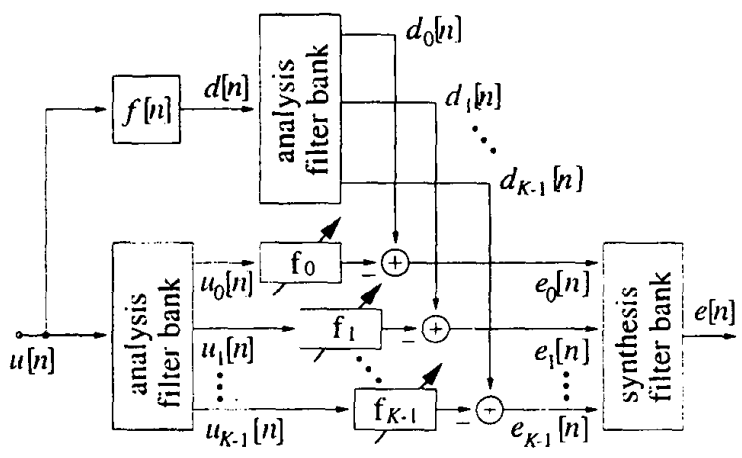

Fig. 5. Constraint projection by subband adaptive system identification.

to the SABs, which will be discussed in Sec. 3.3. Finally by means of using a synthesis bank, a fullband beamformer output can be reconstructed from the SAB outputs.

After adaptation of the SABs, an equivalent fullband beamformer can be calculated by exciting in turn each sensor signal $x_{m}[n]$ by an impulse while feeding zeros to all other inputs. The impulse response observed at the reconstructed beamformer output $e[n]$ then represents the equivalent filter $w_{m}$ in Fig. 1.

\subsection{Subband Projection of Constraints}

Sec. 2.3 highlighted the frequency information incorporated in the constraining vector, f. Therefore, an appropriate projection of $\mathbf{f}$ into the subband domain is required prior to performing $\mathrm{SAB}$ according to Sec. 3.2. This projection can be interpreted as a subband system identification task, which can be evaluated adaptively according to Fig. 5. The impulse response of the unknown system in this identification is given by the coefficients of the constraining vector $\mathbf{f}$, while 
the subband adaptive filters in the adapted state represent the subband constraining vectors, $\mathbf{f}_{m}$. An alternative method to adaptive identification is the direct. analytical projection according to [12], which gives the optimum subband responses $\mathbf{f}_{\boldsymbol{m}}$.

The constraint projection opens an interesting argument why critically sampled systems would fail in this situation. The structure in Fig. 2 may work even if the input signal contained aliasing (as opposed to a multichannel algorithm with explicit desired signal). However, a projection of the constraints would remain suboptimal, as the identification problem in Fig. 5 required either adaptive cross-terms between at least adjacent subbands [3] or the introduction of spectral gaps in the analysis bank [14]. In the first case. the adapted cross terms indicate that aditional beamformers over the $K$ used in Fig. 4 where required, where the latter possibility introduces loss. Hence oversampled filter banks appear to be the correct way forward.

\subsection{Computational Complexity}

For GSC beamforming, the complexity in the nonadaptive preprocessing (4) and (5) requires $L M(L M-$ $r+1)$ multiplications, which is one order of $L$ abovc the processing power for L.MS-type algorithms and only slightly less than what is demanded by the RLS. Note, that therefore regardless of the algorithm. a GSC beamformer yields a complexity of $\mathcal{O}\left(L^{2}\right)$ multiplications. In subband processing, the filter length $L_{\mathrm{SAB}}$ and number of constraints $r_{S A B}$ can be reduced to

$$
L_{\mathrm{SAB}}=\frac{L+L_{p}}{N}, \quad r_{\mathrm{SAB}}=\frac{r+L_{p}}{N}
$$

for a beamformer of identical modelling capabilities to the fullband [11]. If the equivalent fullband beamformer length $L$ is considerably larger than the length of the analysis filters, $L_{p}$, the resulting reduction in computational complexity for going from a fullband to a subband implementation is approximately $N^{3} / K$.

\section{Simulation Results}

The proposed subband adaptive beamformer is compared to a fullband beamformer of equivalent modelling capabilites for the following scenario. An array of $M=11$ sensors receives a white signal of interest from broadside. This signal is corrupted by observation noise at $5 \mathrm{~dB}$ SNR and an interferer illuminating the array from an angle of $v=-20^{\circ}$ at $-37 \mathrm{~dB}$ SINR with spectral proporties as given in Fig. 6 .

Both interference and noise should be adaptively suppressed by a fullband beamformer with $L=100$

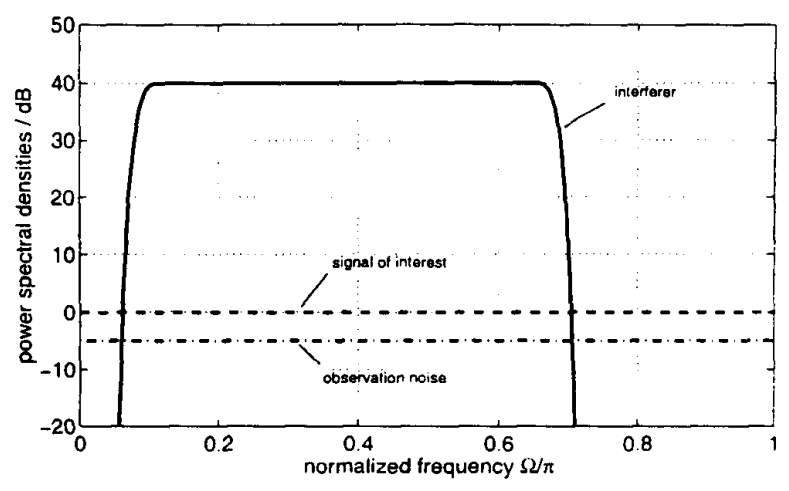

Fig. 6. PSDs of signal components at array.

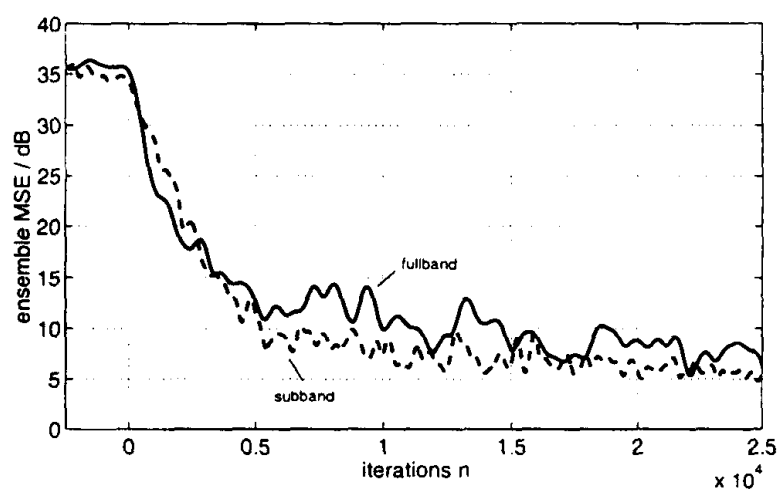

Fig. 7. Learning curves of beamformers.

coefficients in temporal dimension, using a GSC structure updated by the NLMS algorithm with $\tilde{\mu}=0.25$. The SAB works in $K / 2=8$ subbands decimated by $N=14$. With a filter length of $L_{p}=448$ for the prototype filter of the analysis filter bank, the filter length of each of the sub-beamformers in Fig. 4 is $L_{\mathrm{SAB}}=40$ according to (10). The prototype filter is chosen such that the performance limitations of the $\mathrm{SAB}$ due to aliasing in the subbands are below the imposed limitation by the observation noise.

The ensemble averaged squared residual error (beamformer output minus signal of interest) is given for both fullband and subband simulations in Fig. 7 . Although the fullband algorithm initially converges slightly faster and the SAB exhibits a lower steady state error, the performances of both beamformers appear comparable. However, the subband adaptive beamformer requires only $16 \%$ of the calculations used by the fullband beamformer.

After adaptation of the $\mathrm{SAB}$ and reconstruction of an equivalent fullband beamformer according to Sec. 3.2. Fig. 8 shows the calculated beam pattern over 


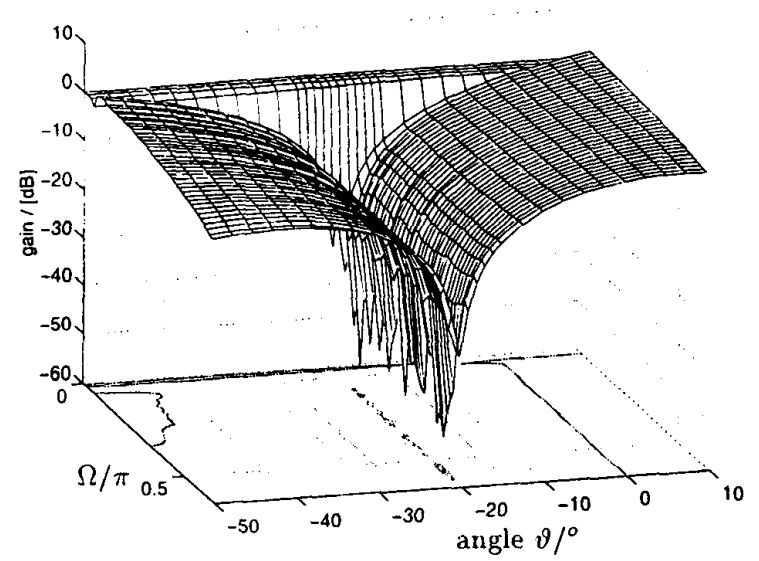

Fig. 8. Beam pattern.

the normalized frequency range $[0 ; 0.65 \pi]$. Note that spatial nulls have been placed at an angle of $\vartheta=-20^{\circ}$ to suppress the interferer present on the frequency interval is shown in Fig. 6. The fulfillment of the constraint to receive the signal of interest from broadside, $\vartheta=0^{\circ}$, can also be verified.

\section{Conclusions}

We-have introduced a computationally efficient broadband beamforming structure, whereby linearly constrained minimum variance beamformer units are operated independently in oversampled subbands. Methods for correctly translating the beamformer's constraints into the subband domain have been discussed. Specifically, we have applied a generalised sidelobe canceller, which allows an unconstrained optimisation using standard adaptive filters. For long beamforming filters, the reduction in computational complexity was shown to be reduced by a factor of approximately $N^{3} / K$, where $K$ is the number of subbands decimated by a factor of $N$.

Limitations of the subband adaptive beamforming (SAB) structure by aliasing and non-perfect reconstruction of the filter banks has been briefly discussed, which however can be controlled by appropriate design of the filter banks. This fact taken into account, similar behaviour with respect to adaptation speed and steadystate performance for a GSC beamformer driven by an NLMS adaptive algorithm has been demonstarted for fullband and subband implementations. The comparable performance of the SAB was however achieved at a much lower computational cost.

\section{Acknowledgements}

S. Weiss, R.W. Stewart, and M. Schabert would like to gratefully acknowledge financial support from DERA,
Malvern, UK.

(c) Crown Copyright 1999 Defence Evaluation and Research Agency UK. This work was carried out as part of Technology Group TG9 of the MoD Corporate Research Programme.

\section{References}

[1] R.T. Compton. "The Relationship Between Tapped Delay-Line and FFT Processing in Adaptive Arrays". IEEE Trans Antennas Propagation, Vol.36(No.1):pp.15-26. Jan. 1988

[2] O.L. Frost, III. "An Algorithm for Linearly Constrained Adaptive Array Processing". Proc. IEEE. Vol.60(No.8):pp.926-935. Aug. 1972.

[3] A. Gilloire and M. Vetterli. "Adaptive Filtering in Subbands with Critical Sampling: Analysis. Experiments and Applications to Acoustic Echo Cancelation". IEEE Trans Signal Processing, Vol.SP40(No.8):pp.1862-1875. Aug. 1992.

[4] L.J. Griffith and C.W. Jim. "An Alternative Approach to Linearly Constrained Adaptive Beamforming". IEEE Trans Antennas Propagation, Vol.30(No.1):pp.27-34, Jan. 1982.

[5] S. Haykin. Adaptive Filter Theory. Prentice Hall, 2nd ed., 1991.

[6] M. Joho and G.S. Moschytz. "On the Design of the Target-Signal Filter in Adaptive Beamforming". IEEE Trans Circuits Systems II, Vol.46(No.7):pp.963-966, July 1999.

[7] W. Kellermann. "Analysis and Design of Multirate Systems for Cancellation of Acoustical Echoes". In Proc. ICASSP, vol 5, pp.2570-2573, New York, 1988.

[8] T.I. Laakso, V. Välimäki, M. Karjalainen, and U.K. Laine. "Splitting the Unit Delay". IEEE Signal Processing Magazine, Vol.13(No.1):pp.30-60, Jan. 1996.

[9] B.D. Van Veen and K.M. Buckley. "Beamforming: A Versatile Approach to Spatial Filtering". IEEE Acoustics, Speech, Signal Processing Magazine, Vol.5(No.2):pp.4-24, April 1988.

[10] G. Wackersreuther. "Some New Aspects of Filters for Filter Banks". IEEE Trans Acoustics, Speech, Signal Processing, Vol. ASSP-34(No.10):pp.1182-1200, Oct. 1986

[11] S. Weiss and R.W. Stewart. On Adaptive Filtering in Oversampled Subbands. Shaker Verlag, Aachen, 1998.

[12] S. Weiss and R.W. Stewart. "On the Optimality of Subband Adaptive Systems". In IEEE Workshop Applications Signal Processing to Audio and Acoustics, pp.59-62, New Paltz, NY, Oct. 1999.

[13] S. Weiß, R.W. Stewart, A. Stenger, and R. Rabenstein. "Performance Limitations of Subband Adaptive Filters". In Proc. EUSIPCO, vol. III, pp.1245-1248, Rhodos, Greece, Sept. 1998.

[14] Y. Yamada. H. Ochi, and H. Kiya. "A Subband Adaptive Filter Allowing Maximally Decimation". IEEE Journal Selected Areas Communications. Vol.12(No.9):pp.1548-1552, Sept. 1994. 